\title{
Vertical transport and tunnelling in rare-earth nitride heterostructures
}

\author{
Jackson D. Miller, Felicia H. Ullstad, H. Joe Trodahl, Ben. J. Ruck and Franck Natali \\ The MacDiarmid Institute for Advanced Materials and Nanotechnology, School of Chemical and \\ Physical Sciences, Victoria University of Wellington, P.O. Box 600, Wellington 6140, New Zealand \\ E-mail: franck.natali@vuw.ac.nz
}

\begin{abstract}
We report an investigation of the ferromagnetic semiconductor rare earth nitrides (RENs) for their potential for cryogenic-temperature electronics and spintronics application. We have indentified ohmic contacts suitable for the device structures that demand electron transport through interface layers, and grown REN/insulator/REN heterostructures that display tunnelling characteristics, an enormous $400 \%$ tunneling magnetoresistance and a hysteresis promising their exploitation in non-volatile magnetic random access memory.
\end{abstract}

Keywords: rare earth nitrides, ferromagnetic semiconductors, ohmic contact, magnetic tunnel junctions, memory elements

\section{Introduction}

It is now well-established that the rare earth nitrides (RENs) form a series of intrinsic ferromagnetic semiconductors suitable for full exploitation of the coupled magnetic/electronic technology, so-called spintronics [1]. They display their magnetic properties only at low temperatures $(<70 \mathrm{~K})$ and while it prevents room temperature applications, they are materials of choice on the more demanding route to devices for cryogenic electronics [2]. Their complementary properties, in particular the varied set of magnetic properties across the series, provide huge scope for use in memory elements such as non-volatile magnetic random access memory (MRAM) to support superconducting and quantum computing [3-10]. In addition, a strong impetus to select RENs over ferromagnetic metals or diluted magnetic semiconductors (DMS) derives from the ability to dope the materials without losing ferromagnetic spin alignment $[11,12]$. This offers potential integration and impedance matching with the materials and processing for superconducting electronics. However, only little has been reported concerning the study of vertical transport, the so- called Current Perpendicular to Plane (CPP) geometry, [1316] with the literature focussing mostly on electrical transport properties in planar structures for fundamental studies [1721]. Since devices will mostly require electronic transport across material interfaces, there is an urgent need to provide a general guideline for the design and analysis of vertical tunnelling devices and heterostuctures if the RENs are to be technologically exploited. Remaining hurdles like ensuring ohmic contacts, developing lithographic procedures and reducing device size towards a practical $1 \mu \mathrm{m}^{2}$ target have to be overcome. Progress towards all of these goals are presented and discussed in this paper. In particular, we show evidence for ohmic contact to $\mathrm{GdN}$, the prototypical REN, in CPP device geometries, and optically defined device areas down to $10 \times 10 \mu \mathrm{m}^{2}$ without oxidation of the GdN layer. Finally a clear hysteresis at low magnetic field is oberved in magnetic tunnelling junctions, a promising advance towards the development of REN-based memory element structures.

\section{Methods}

In this study, heterostuctures consisting of metal/GdN/metal thin layers and magnetic tunnelling devices 
consisting of metal/REN/insulator/REN/metal thin layers have been fabricated using a multi-step approach on $300 \mathrm{~nm}$ thick $\mathrm{SiO}_{2}$ layers thermally grown on silicon (100) wafers. The metal/GdN/metal structures were used as a model system to determine the nature of the contact, ohmic or Schottky, between a range of metallic layers, here $\mathrm{Al}, \mathrm{Au}$ and $\mathrm{Gd}$, and the GdN semiconductor layer. Due to their wide band gap and their chemical and structural compatibility with the RENs, $\mathrm{GaN}$ or AlN are used as insulating layers to form tunnel barriers. We have chosen $\mathrm{GdN}$ and $\mathrm{SmN}$ among the fourteen RENs due to their contrasting magnetic properties $[1,16]$.

RENs have been recognized for decades to react rapidly in air, requiring careful capping. Within this scenario REN-based CPP structures require special attention to avoid oxidation from open edges exposed to air that then slowly react and degrade the device performances. Figure 1 schematically shows the fabrication steps we developed to avoid the problem. Special attention was given, based on a so-called caisson-like structure [Figure 1(a)], both to overcome the oxidation problem and to reduce the size of devices from the $100 \times 200 \mu \mathrm{m}$ that we have previously achieved [16]. Our tunnelling devices are defined by small apertures (caisson) in a GaN layer that expose a segment of an underlying bottom metal contact. The stack then consists of the bottom metal contact strip (i.e. $\mathrm{Al}, \mathrm{Au}$ or $\mathrm{Gd}$ ), a $40 \mathrm{~nm}$ thick $\mathrm{GaN}$ insulator (with square caissons of 10, 20, 30 or $40 \mu \mathrm{m}$ ), the tunnelling stack (i.e. GdN/GaN/GdN or GdN/AlN/SmN) and top metal contact (i.e. Al, Gd); see Figure 1(b). The REN/top metal contact stack (step 3) fills the active area, allowing direct contact between the metallic bottom contact (step 1) and the stack. The GaN layer that defines the caisson (step 2) is grown thinner than the stack to ensure layer continuity across the active area edge. The surface profile across a GaN caisson region is shown in Figure 1(c). The two steep slopes at 600 and $640 \mu \mathrm{m}$ are the caisson edges, so that the $35 \mathrm{~nm}$ dip in the middle shows the active area window depression that defines the device. Up to 20 lithographically defined tunnelling devices were prepared on a single chip, thus providing numerous devices of various sizes in a single preparation.

The REN (SmN, GdN), insulating ( $\mathrm{GaN}, \mathrm{AlN})$ and top metal contact ( $\mathrm{Al}, \mathrm{Gd})$ layers were grown at ambient temperature in a molecular beam epitaxy system with a base pressure $<10^{-9}$ Torr. The ambient growth temperature allows GdN and SmN layers to be smooth and strongly (111) textured. Au was used only as a bottom contact layer and was grown in a different physical vapor evaporator. Further details regarding the growth conditions (deposition rate, nitrogen precursor, etc) for the REN and insulating layers can be found elsewhere $[16,18]$.
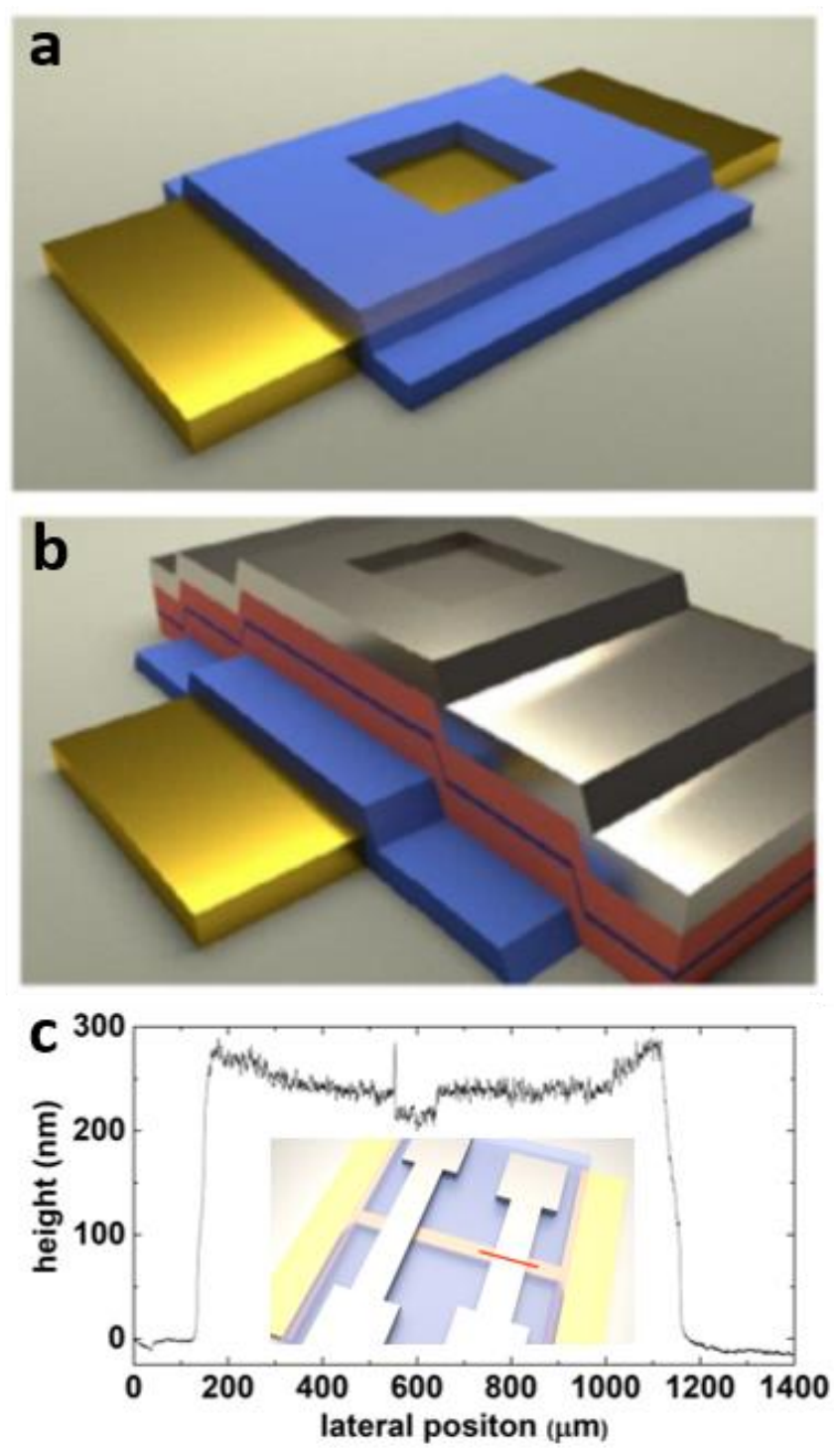

Figure 1. Schematic representation of the fabrication steps; (a) GaN caisson-like structure (blue) grown over the metal bottom contact (yellow) and (b) entire structure with two REN layers (red) separated by the tunnel barrier (black) and finished with the top contact (grey). (c) Surface profile of the stack in a metal/REN/insulator/REN/metal structure along the red line shown in the inset. The dip in the middle of the surface profile is where the stack is grown on top of the active area caisson.

The current/voltage (I/V) characteristics of the structures were measured between the crossed metallic strips, each offering separate voltage and current leads. The (I/V) measurements of the devices were performed between ambient temperature and $2 \mathrm{~K}$ in both a simple closed-cycle cryostat and a Quantum Design Physical Property Measurement System (PPMS) with the facility to apply magnetic fields to $9 \mathrm{~T}$. Care was taken to ensure that the metal strips were sufficiently conductive to form virtual equipotentials, so that in turn the current densities through the 
devices were uniform, as was established by noting that the net current in most devices was proportional to the caisson area. To ease comparison of structures with different areas we report the results below in terms of the current density $(J)$ rather than the measured net current.

\section{Results}

\subsection{Metal/GdN/Metal structures}

In view of the propensity for Schottky barrier formation across metal-semiconductor interfaces it is important to establish that the measured impedances of the tunnelling structures are dominated by the very thin tunnelling barrier layer, rather than the metal/GdN interfaces. We have thus investigated several structures in which the barrier layer was omitted to determine the resistance of metal/GdN/metal stacks. For technical convenience we have investgated contacts comprising $\mathrm{Al}, \mathrm{Au}$ and $\mathrm{Gd}$.

Turning first to Al contacts, there is no sign of the nonlinearity commonly associated with Schottky barriers, and the linear resistance observed yields a resistivity of the $100 \mathrm{~nm}$ thick GdN layer of $\sim 3 \Omega$.cm at $4 \mathrm{~K}$ (not shown). That value is within the range that is commonly measured for in plane $\mathrm{GdN}$ layers, charactersitic of a carrier density of order $10^{19} \mathrm{~cm}^{-3}$ $[12,21]$. The impedance ratio (voltage/current density; V/J) provides a direct estimate of the impact the $\mathrm{GdN}$ resistance has on the tunnelling studies below. The data give $\mathrm{V} / \mathrm{J} \sim 10^{-4}$ $\Omega . \mathrm{cm}^{2}$ at low temperatures, which will be seen below to be six orders of magnitude smaller than the impedance ratio found in the tunnelling structures.

We have previously reported that $\mathrm{Au}$ forms an ohmic contact in measurements of in-plane transport [22], a geometry which is less sensitive to Schottky barrier effects due to its larger form factor (length/area), as we have also cofirmed in the present geometry [16]. Clearly both $\mathrm{Al}$ and $\mathrm{Au}$ form ohmic contacts to GdN.

The assessment of Gd metal as a possible contact material is critical as the exchange interaction with $\mathrm{GdN}$ or $\mathrm{SmN}$ across the material interface provides the pinning of the GdN magnetisation allowing observation of switching behaviour in a magnetic tunnnelling junction [16]. Similarly to the case of $\mathrm{Al}$ and $\mathrm{Au}, \mathrm{a} \mathrm{Gd} / \mathrm{GdN} / \mathrm{Gd}$ device structure was studied. As can be seen in Figure 2, the $\mathrm{J} / \mathrm{V}$ plot is linear at ambient temperature but develops a modest non-linearity at low temperature. The impedance ratio $(\mathrm{V} / \mathrm{J}) \sim 3 \times 10^{-3} \Omega . \mathrm{cm}^{2}$ at 4 $\mathrm{K}$ is a factor of $10^{2}$ larger than $\mathrm{Au} / \mathrm{GdN} / \mathrm{Al}$ structures. The larger resistance suggests the formation of Schottky barriers at the $\mathrm{Gd} / \mathrm{GdN}$ interface which substantially deplete the carrier concentration within GdN. The non-linearity of the lower temperature is also an indication of a tunnelling-mode transport across the interfaces of the structure. While the conduction mode is not ohmic, the specific resistance remains three orders of magnitude smaller than the minimum impedance ratio across the tunnelling barrier structure we will see below, thus permitting the use of metallic Gd as a contact material.

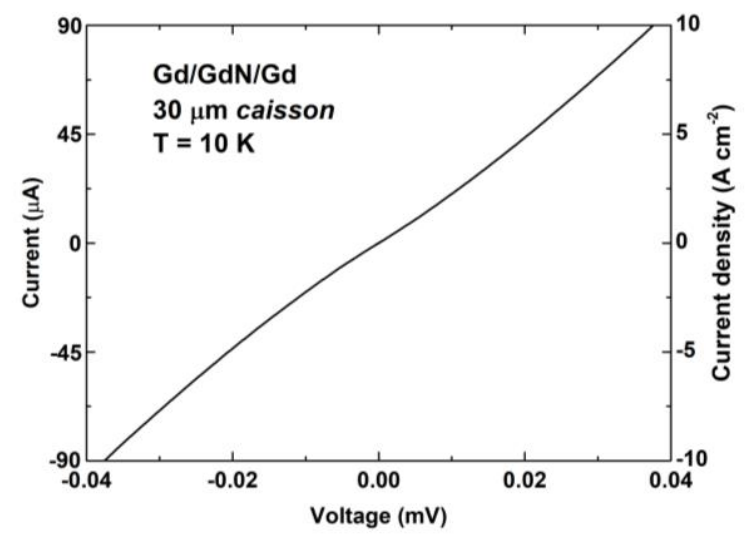

Figure 2. I/V and J/V characteristics of a Gd/GdN/Gd structure at $10 \mathrm{~K}$ showing only weak nonlinearity.

\subsection{Tunnelling through engineered barriers}

In Figure 3 we show the strongly nonlinear response of a $\mathrm{Au} / \mathrm{GdN} / \mathrm{GaN} / \mathrm{SmN} / \mathrm{Al}$ structure with target layer thicknesses of $100 \mathrm{~nm} \mathrm{GdN}$ and $\mathrm{SmN}$ and $3 \mathrm{~nm} \mathrm{GaN}$. Even at the largest voltage, $0.9 \mathrm{~V}$, the impedance ratio of order $6 \times 10^{-2} \Omega . \mathrm{cm}^{2}$ is still almost three orders of magnitude larger than that from the device without an engineered barrier. The data of Figure 3 have thus been fitted to the Simmons model [23,24] to yield estimates of $\sim 1 \mathrm{eV}$ and $\sim 2 \mathrm{~nm}$, respectively, for the $\mathrm{GaN}$ barrier height and width. These are within the range expected for the $3.3 \mathrm{eV}$ band gap of $\mathrm{GaN}$ and the designed $2.5 \mathrm{~nm}$ barrier thickness. There is an asymmetry as expected with the $\mathrm{GdN} / \mathrm{SmN}$ asymmetry of the source/drain for the tunnelling current.

Figure 3. $\mathrm{I} / \mathrm{V}$ and $\mathrm{J} / \mathrm{V}$ characteristics of a $60 \times 60(\mu \mathrm{m})^{2}$

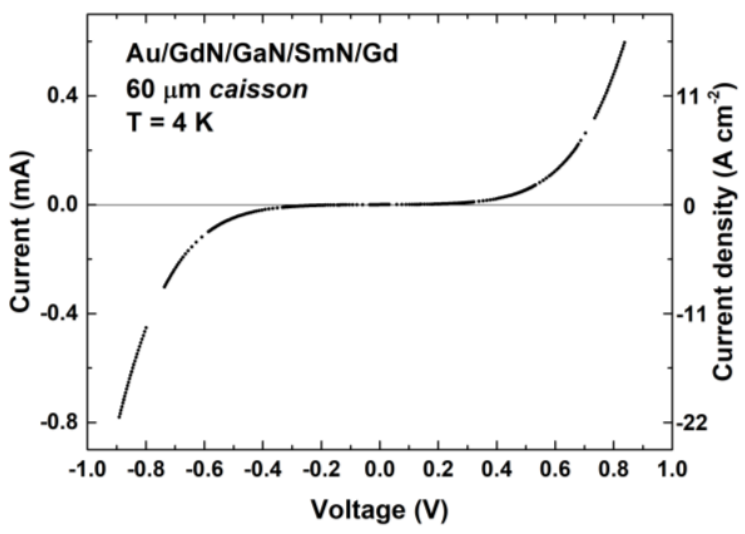

$\mathrm{Au} / \mathrm{GdN} / \mathrm{GaN} / \mathrm{SmN} / \mathrm{Al}$ structure at $4 \mathrm{~K}$. 


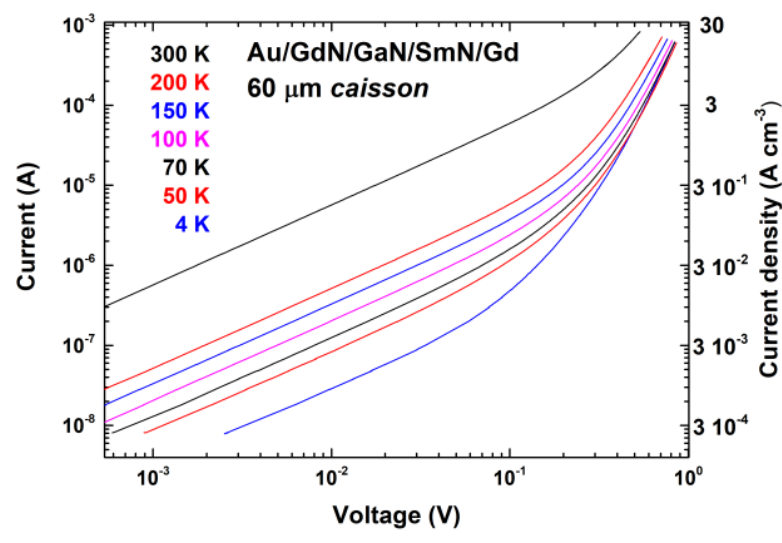

Figure 4. Temperature-dependent $\mathrm{I}-\mathrm{V}$ and $\mathrm{J} / \mathrm{V}$ characteristics of the device in Figure 3. These data were collected under a positive applied voltage, with current flowing from GdN to SmN.

The temperature dependant I/V response of the forwardbiased device is shown at temperatures up to $300 \mathrm{~K}$ in Figure 4 . The response is again seen to be strongly nonlinear, yielding Simmons-model estimates of $\sim 1 \mathrm{eV}$ and $\sim 2 \mathrm{~nm}$ for the barrier height and width that are at most only weakly temperature dependent. The data show a linear behaviour at low voltage, where the conductance increases monotonically with temperature. In contrast the nonlinear conduction at larger applied voltage shows a complex temperature dependence, signalling clearly the shifting band edges across the Curie temperatures of $\mathrm{GdN}\left(\mathrm{T}_{\mathrm{C}} \sim 70 \mathrm{~K}\right)$ and $\mathrm{SmN}\left(\mathrm{T}_{\mathrm{C}} \sim 30 \mathrm{~K}\right)$ [1].

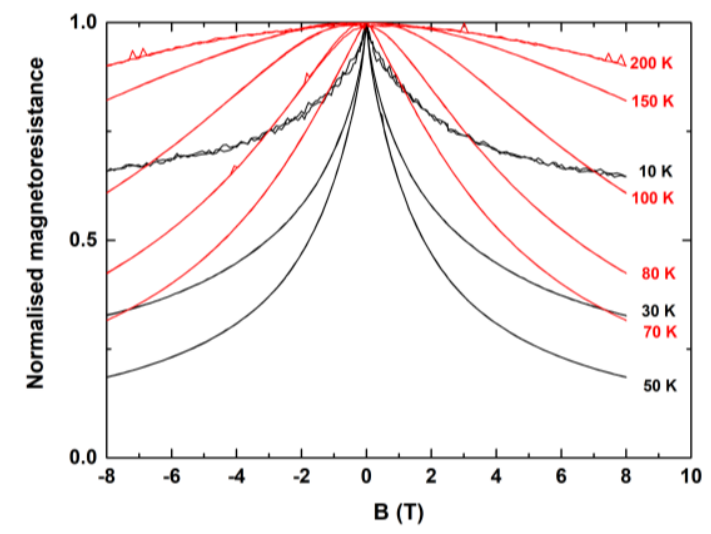

Figure 5. Tunnelling magnetoresistance for the device of Figures 3 and 4 at different temperatures, $1 \mu \mathrm{A}$ excitation.

The influence of spin alignment in the GdN and SmN layers is seen in the magnetic-field control of resistance of Figure 5. Note that the conventional characterisation of tunneling magnetoresistance is quoted as

$$
\mathrm{TMR}=[R(B)-R(\max )] / \mathrm{R}(\min )),
$$

where $R(\max )$ and $\mathrm{R}(\min )$ are the maximum and minimum resistance of the device, close to 0 Tesla and 8 Tesla in the present case. Thus the five-fold reduced resistance at $50 \mathrm{~K}$ corresponds to a TMR of $400 \%$.

Of more direct interest for memory element devices is to establish the tunneling resistance in the very low-field region, where hysteresis exerts an influence. Thus in Figure 6 the hysteresis is displayed at $2 \mathrm{~K}$, plotted as

$$
\delta \mathrm{R}=\left[\mathrm{R}_{\mathrm{up}, \mathrm{down}}(B)-\mathrm{R}_{\text {ave }}(B)\right] \equiv \pm\left[\mathrm{R}_{\mathrm{up}}(B)-\mathrm{R}_{\text {down }}(B)\right] / 2,
$$

where $R_{\text {up }}$ and $R_{\text {down }}$ are the resistances under increasing and decreasing magnetic fields, respectively. It is expected that the relative spin alignments on the two sides of the barrier influence the measured resistance, and indeed here there are evident transitions near the coercive fields of GdN $(\sim 0.02 \mathrm{~T})$ and $\mathrm{SmN}(>2 \mathrm{~T})$ [1]. The switching shown in Figure 6 persists up to $30 \mathrm{~K}$, the $\mathrm{T}_{\mathrm{c}}$ of $\mathrm{SmN}$, demonstrating that the hysteresis behaviour requires both the GdN and SmN layers to be in their ferromagnetic states.

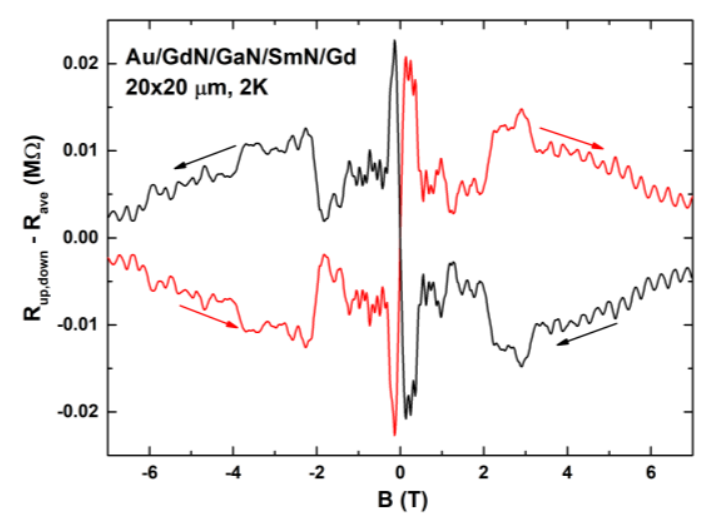

Figure 6. Magnification of low field hysteretic behaviour for an $\mathrm{Au} / \mathrm{GdN} / \mathrm{AlN} / \mathrm{SmN} / \mathrm{Al}$ structure.

\section{Summary}

We have reported growth of thin films of ferromagnetic semiconducting rare-earth nitrides in layered structures, designed for the investigation of the electronic reponse in current perpendicular to plane (CPP) configurations. Special care was taken to ensure that the devices were small enough to show excellent CPP impedance.

The work identified three contact metals ( $\mathrm{Au}, \mathrm{Al}, \mathrm{Gd})$ and investigated their susceptability to Schottky-barrier problems. Contacts comprising Au and $\mathrm{Al}$ show ohmic behaviour, while there is evidence of a depressed carrier concentration in $\mathrm{GdN}$ at an interface with $\mathrm{Gd}$.

Identification of suitable ohmic-contact metals facilitated investigation of structures in which insulating tunnel barriers, 1-2 nm of AlN or GaN, were introduced between two REN 
layers, thus forming a magnetic tunnel junction. The structures showed clear negative tunnelling magnetoresistance as large as $400 \%$ (i.e. a contrast factor of 5) between fields of 0 and 9 T.

Finally the work has demonstrated clear tunnellingresistance hysteresis, opening the door to devloping tunneling magnetoresistance memory for cryogenic memory banks.

\section{Acknowledgements}

We acknowledge funding from the Marsden Fund (Grant No.13-VUW-1309), the New Zealand Endeavor fund (Grant No. RTVU1810) and the MacDiarmid Institute for Advanced Materials and Nanotechnology, funded by the New Zealand Centres of Research Excellence Fund. Jackson Miller thanks the School of Chemical and Physical Sciences for financial support.

\section{References}

[1] Natali F, Ruck B J, Plank N O V, Trodahl H J, Granville S, Meyer C and Lambrecht W R L 2013 Prog. Mater. Sci. 581316.

[2] IRDS Roadmap Cryogenic Electronics and Quantum Information Processing 2018.

[3] Birge N O, Madden A E and Naaman O 2018 Proc. SPIE $10732107321 \mathrm{M}$.

[4] Herr Q P, Herr A Y, Oberg O T and Ioannidis A G $2011 \mathrm{~J}$. Appl. Phys. 109103903.

[5] Mukhanov O A 20111 IEEE Trans. Appl. Superconductivity 2760.

[6] Holmes D S, Ripple A L and Manheimer M A 2013 IEEE Trans. Appl. Superconductivity 23, 1701610.

[7] Manheimer M A 2015 IEEE Trans. Appl. Superconductivity 251.
[8] 4834.

[9] Tolpygo S K, Bolkhovsky V, Weir T, Wynn A, Oates D, Johnson L and Gouker M 2016 IEEE Trans. Appl. Supercond 261.

[10] Johnson M W, Bunyk P, Maibaum F, Tolkacheva E, Berkley A J, Chapple E M, Harris R, Johansson J, Lanting T, Perminov I, Ladizinsky E, Oh T and Rose G Supercond. Sci. Technol. 201023065004.

[11] Lee C -M, Warring H, Vézian S, Damilano B, Granville S, Khalfioui M A, Cordier Y, Trodahl H J, Ruck B J and Natali F 2015 Appl. Phys. Lett. 106022401

[12] Trodahl H J, Natali F, Ruck B J and Lambrecht W R L Phys. Rev. B 96115309.

[13] Mudali P H, Pal A and Blamire M G 2014 Phys. Rev. B 89, 094414.

[14] Senapati K, Blamire M G and Barber Z H 2011, Nature Materials 10, 849.

[15] Pal A, Senapati K, Barber Z H and Blamire M G 2013, Adv. Mat. 25, 5581.

[16] Warring H, Trodahl H J, Plank N O V, Natali F, Granville S and Ruck B J 2016 Phys. Rev. Appl. 6044002.

[17] Scarpulla M, Gallinat C, Mack S, Speck J and Gossard A 2009 J. Cryst. Growth 311 1239-1244.

[18] Natali F, Plank N, Galipaud, J, Ruck B, Trodahl H, Semond F, Sorieul S and Hirsch L 2010 J. Cryst. Growth 3123583.

[19] Senapati K, Fix T, Vickers M E, Blamire M G and Barber Z H 2011 Phys. Rev. B 83014403.

[20] Wachter P 2012 Res. Phys. 2012290.

[21] Plank N O V, Natali F, Galipaud J, Richter J H, Simpson M, Trodahl H J and Ruck B J 2011 Appl. Phys. Lett. 98112503.

[22] Ullstad F, Chan J R, Warring H, Plank N, Ruck B, Trodahl J and Natali F 2015 AIMS Mat. Sc. 279.

[23] J. G. Simmons 1963, J. Appl. Phys. 34, 1793.

[24] J. G. Simmons 1964, J. Appl. Phys. 35, 2655. 